\title{
GRP78 Regulates Apoptosis, Cell Survival and Proliferation in 5-Fluorouracil-resistant SNUC5 Colon Cancer Cells
}

\author{
JUN HEE LEE ${ }^{1}$, YEO MIN YOON ${ }^{2}$ and SANG HUN LEE ${ }^{2,3}$ \\ ${ }^{1}$ Department of Pharmacology and Toxicology, University of Alabama School of Medicine, Birmingham, AL, U.S.A.; \\ ${ }^{2}$ Medical Science Research Institute, Soonchunhyang University Hospital, Seoul, Republic of Korea; \\ ${ }^{3}$ Department of Biochemistry, Soonchunhyang University College of Medicine, Cheonan, Republic of Korea
}

\begin{abstract}
Fluorouracil (5-FU) is an effective anticancer drug. However, the development of drug resistance has limited its chemotherapeutic efficacy. To address this problem, we investigated the expression of glucose-regulated protein (GRP78, $78 \mathrm{kDa}$ ) in 5-FU-resistant colorectal cancer (CRC) cells (SNUC5/5FUR). GRP78 was highly expressed in the SNUC5/5FUR cells compared to wild-type SNUC5 cells. In the presence of 5-FU, GRP78 knockdown induced apoptosis via activation of caspase-3 and poly(ADP-ribose)polymerase 1. GRP78 also inhibited the production of intracellular reactive oxygen species by regulating stressassociated signaling pathways. Furthermore, GRP78 enhanced cell survival and proliferation via activation of the phosphatidylinosito-3-kinase-AKT-mammalian target of rapamycin axis and cell cycle-associated proteins. These effects were blocked upon GRP78 knockdown, which indicates that GRP78 is involved in the development of 5-FU resistance in these CRC cells. Therefore, a combination of chemotherapy and GRP78-specific targeting may counteract 5-FU resistance in CRC cells.
\end{abstract}

Colorectal cancer (CRC) is the second leading cause of cancer-related deaths in men and the third leading cause in women (1). Despite improvements in surgical techniques, development of chemotherapy, and molecular targeting therapy, cancer treatment remains a therapeutic challenge. Additionally, drug resistance is a major factor that limits the effectiveness of chemotherapy, which is the main therapeutic strategy for cancer treatment (2). Potential mechanisms of drug resistance include increased drug efflux, mutation of

Correspondence to: Sang Hun Lee, Ph.D., Medical Science Research Institute, Soonchunhyang University Hospital, Seoul 04401, Republic of Korea. Tel: +82 027099029, Fax: +82 027925812, e-mail: ykckss1114@ nate.com; jhlee0407@sch.ac.kr

Key Words: 5-Fluorouracil, GRP78, colorectal cancer cells, drug resistance, apoptosis, proliferation. drug targets, defective DNA damage repair, activation of alternative signaling pathways, and evasion from cell death cascades; however, a complete understanding of the mechanisms underlying drug resistance remains unclear (2). Therefore, the discovery and targeting of novel predictive biomarkers has a key role to play in effective CRC therapy.

The 78-kDa glucose-regulated protein (GRP78), also referred to as binding immunoglobulin protein and heatshock protein family A member 5, belongs to the heat-shock protein 70 family and acts as a stress-inducible molecular chaperone (3). GRP78 also regulates various cellular processes, such as translocation of newly synthesized polypeptides across the endoplasmic reticulum (ER) membrane, regulating calcium homeostasis, acting as an ER stress sensor, and targeting misfolded proteins for ERassociated degradation (4). In cancer cells, GRP78 functions as a multifunctional cell-surface receptor that is involved in promoting cell proliferation and viability (5). Moreover, due to its anti-apoptotic effect, GRP78 is induced in a wide variety of cancer cell lines, including drug-resistant ones (68). Notably, GRP78 expression is significantly higher in colon cancer than in colon adenoma and normal tissues (9). GRP78 down-regulation results in colon cancer sensitization to drug-induced apoptosis (10). These observations highlight a potentially important role of GRP78 in cancer therapy.

5-Fluorouracil (5-FU) is widely used as a chemotherapeutic agent in the treatment of a wide variety of cancer types. 5-FU-based therapy significantly increases both the response and survival rates in patients with colon cancer (11). 5-FU causes cytotoxicity in CRC cells via inhibition of thymidylate synthase, incorporation of 5-FU derivatives into DNA, inhibition of RNA synthesis, and genotoxic stressinduced apoptosis (12). Although treatment with 5-FU is beneficial in treating cancer, 5-FU resistance has restricted its therapeutic efficacy in patients with CRC. In order to improve the therapeutic efficacy of 5-FU and elucidate the mechanism(s) underlying the development of 5-FU resistance in CRC cells, we examined the expression of GRP78 in the 5-FU-resistant CRC cell line SNUC5/FUR. In 
addition, we explored whether GRP78 regulates CRC cell survival, proliferation, and apoptosis upon 5-FU treatment and the underlying mechanism of 5-FU resistance through regulation of GRP78-mediated apoptosis and proliferation signal pathway.

\section{Materials and Methods}

SNUC5 culture. Wild-type (SNUC5) and 5-FU-resistant (SNUC5/5FUR) CRC cells were procured from Chosun University (Gwangju, Republic of Korea). The SNUC5 and SNUC5/5FUR cells were cultured in RPMI-1640 medium (Gibco BRL, Gaithersburg, MD, USA) containing $10 \%$ (v/v) fetal bovine serum (FBS, Gibco $\mathrm{BRL})$. Both cell lines were incubated in $5 \% \mathrm{CO}_{2}$ at $37^{\circ} \mathrm{C}$ in a humidified incubator.

Treatment of SNUC5 and SNUC5/5FUR cells. The SNUC5 and SNUC5/5FUR cells were washed twice with phosphate-buffered saline (PBS), and fresh RPMI-1640 medium supplemented with 1\% FBS was added. Apoptosis, cell proliferation, and signaling pathway components were investigated after pretreating the cells with 5-FU $(140 \mu \mathrm{M})$.

Immunochemistry. Untreated SNUC5 and SNUC5/5FUR cells were cultured on glass slides in 24-well plates. Next, the cells were washed three times for $5 \mathrm{~min}$ each with PBS and fixed with $4 \%$ paraformaldehyde (Sigma-Aldrich, St. Louis, MO, USA). Immunofluorescence staining was performed using the primary antibody against GRP78 (Santa Cruz Biotechnology, Santa Cruz, CA, USA) and Alexa-488 secondary antibody (Thermo Fisher Scientific, Waltham, MA, USA). Nuclei were stained with 4',6'-diamidino-2-phenylindole (DAPI; Sigma-Aldrich). The immunostained samples were observed using a confocal microscope (Olympus, Tokyo, Japan).

Western blot assay. Homogenates of treated and untreated SNUC5 and SNUC5/5FUR cells (total protein, $20 \mu \mathrm{g}$ ) were separated using $8-12 \%$ sodium dodecyl sulfate-polyacrylamide gel electrophoresis. Proteins were transferred onto nitrocellulose membranes. After washing the membranes with a solution containing Tris- $\mathrm{HCl}(10 \mathrm{mM}, \mathrm{pH} 7.6)$, $\mathrm{NaCl}(150 \mathrm{mM})$, and Tween $20(0.05 \%)$, the membranes were preincubated with skimmed milk (5\%) for $1 \mathrm{~h}$ at room temperature and then incubated with primary antibodies against GRP78, cleaved caspase-3 (C-caspase-3), cleaved poly(ADP-ribose)-polymerase 1 (CPARP1), p38, phospho-p38 (p-p38), c-JUN N-terminal kinase (JNK), p-JNK, p53, p-p53, ataxia-telangiectasia mutated (ATM), p-ATM, phosphatidylinositol 3-kinase (PI3K), p-PI3K, AKT, p-AKT, mammalian target of rapamycin (mTOR), p-mTOR, cyclin-dependent kinase 2 (CDK2), cyclin E, CDK4, cyclin D1, and $\beta$-actin (Santa Cruz Biotechnology). The membranes were washed three times and the primary antibodies were detected using goat anti-rabbit/anti-mouse IgG conjugated with horseradish peroxidase (Santa Cruz Biotechnology). The protein bands were visualized by enhanced chemiluminescence (GE healthcare, Chicago, IL, USA).

Down-regulation of GRP78 expression by RNA interference. Treated and untreated SNUC5 cells $\left(2 \times 10^{5}\right.$ cells) were seeded in $60-\mathrm{mm}$ dishes and transfected with small interfering RNA (siRNA) in serum-free RPMI-1640 medium (Gibco BRL) using Lipofectamine 2000 , according to the manufacturer's instructions (Thermo Fisher
Scientific). At 48-h post-transfection, total protein was extracted and expression of GRP78 was assessed using western blot analysis. The siRNA used for down-regulating GRP78 (si-GRP78) and the scrambled oligonucleotide sequence (si-scr) were synthesized by Bioneer (Daejeon, Korea).

Flow cytometry. To confirm the expression of GRP78, SNUC5 and SNUC5/5FUR cells were incubated with primary antibody to GRP78 for $1 \mathrm{~h}$ on ice. After thoroughly washing, the cells were incubated with fluorescent dye-conjugated secondary antibody for $30 \mathrm{~min}$ on ice. Next, the cells were washed with PBS. To measure apoptosis, SNUC5/5FUR cells were stained with propidium iodide (PI) and annexin V-fluorescein isothiocyanate (FITC). To investigate the intracellular production of reactive oxygen species (ROS), cells of both lines were stained with dihydroethidium for 30 min at $37^{\circ} \mathrm{C}$. To calculate the proportion of cells in each phase of the cell cycle, cells were stained with PI. Each sample was quantatively analyzed using CyFlow Cube 8 (Sysmex Partec, Münster, Germany) and FSC Express (De Novo Software, Los Angeles, CA, USA).

Statistical analysis. Results were expressed as the mean \pm standard error of the mean (SEM) and analyzed using one-way analysis of variance (ANOVA). For comparing the means of more than three groups, the Bonferroni-Dunn test was used. Data were considered to be significantly different at values of $p<0.05$.

\section{Results}

Expression of GRP78 in 5-FU-resistant CRC cells. To understand the role of GRP78 in the development of drug resistance in cancer cells, we assessed GRP78 expression in wild-type and 5-FU-resistant SNUC5 CRC cells. Western blot analysis revealed that the level of GRP78 was significantly higher in SNUC5/5FUR cells than in SNUC5 cells (Figure 1A). Flow cytometric analysis showed that the percentage of GRP78-positive cells was significantly higher in SNUC5/5FUR cells than in SNUC5 cells (Figure 1B). Immunofluorescence staining also showed that the expression of GRP78 was significantly higher in SNUC5/5FUR cells than in SNUC5 cells (Figure 1C). These results confirmed high GRP78 expression in the 5-FU-resistant CRC cells.

Effect of GRP78 on apoptosis in CRC cells. It has been reported that the level of GRP78 is high in several cancer cell lines and that it regulates the balance between survival and apoptosis in these cells (3). To understand whether GRP78 regulates apoptosis in CRC cells treated with an anticancer drug, we measured apoptosis and GRP78 expression in SNUC5/5FUR cells. The level of GRP78 significantly decreased in the SNUC5/5FUR cells upon transfection with si-GRP78 (Figure 2A).

After treatment of SNUC5/5FUR cells with 5-FU, the levels of C-caspase-3 and C-PARP1 were analyzed using western blot analysis (Figure 2B). The levels of C-caspase3 and C-PARP1 significantly increased in the SNUC5/5FUR 
A

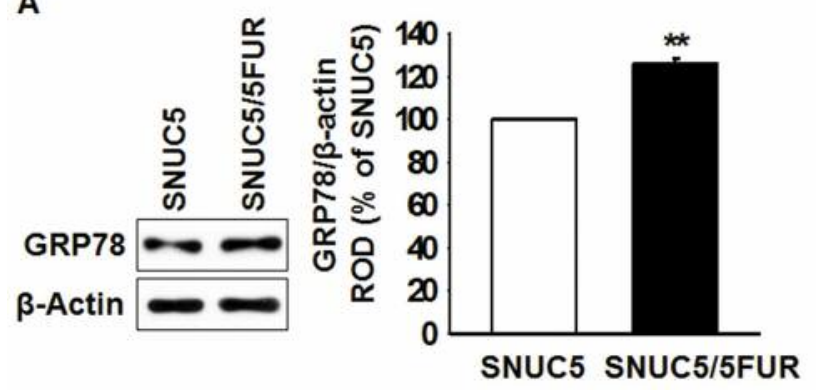

B

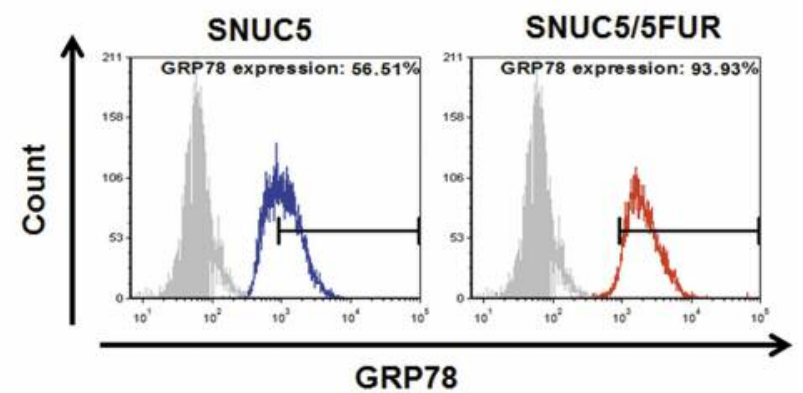

C

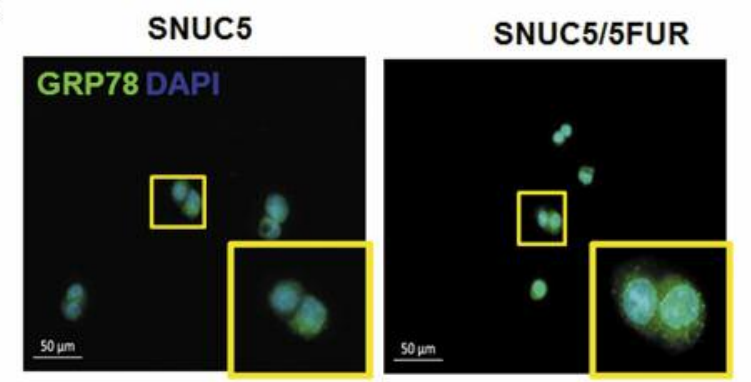

Figure 1. Glucose-regulated protein (GRP78) levels in wild-type (SNUC5) and 5-fluorouracil-resistant (SNUC5/5FUR) colorectal cancer cells. A: Western blots showing GRP78 expression in the SNUC5 and SNUC5/FUR cells. The expression of GRP78 was normalized relative to that of $\beta$-actin. Values represent the mean \pm standard error of the mean. *Significantly different at $p<0.01$ compared SNUC5 cells. B: Flow cytometric analysis of GRP78 expression in SNUC5 and SNUC5/5FUR cells. The grey line indicates negative control. The blue and red lines indicate GRP78 stained with antibody to GRP78. C: Immunofluorescence staining to detect GRP78 (green) in SNUC5 and SNUC5/5FUR cells. 4',6'-Diamidino-2-phenylindole (DAPI, blue) was used for nuclear staining. Scale bar $=50 \mu \mathrm{m}$.

cells treated with 5-FU compared to those treated with PBS (Figure 2C). In addition, GRP78 knockdown significantly increased the expression of C-caspase-3 and C-PARP1 in SNUC5/5FUR cells treated with and without 5-FU treatment (Figure 2C). Furthermore, flow cytometric analysis using PI and annexin V-FITC staining revealed that knockdown of GRP78 induced significant apoptosis of SNUC5/5FUR cells upon treatment with 5-FU (Figure 2D and E). These data suggest that the level of GRP78 regulates 5-FU-induced apoptosis of drug-resistant CRC cells.

GRP78 regulates $5-F U$-induced oxidative stress in drugresistant CRC cells. Excessive ROS production induces apoptosis (13). We evaluated the effect of GRP78 on ROS production in the drug-resistant $\mathrm{CRC}$ cells upon 5-FU treatment using flow cytometry. After treatment with 5-FU for $24 \mathrm{~h}$, ROS production significantly increased in SNUC5/5FUR cells transfected with si-GRP78 compared to those transfected with si-scr (Figure 3A). To confirm whether GRP78 mediates the phosphorylation of oxidative stressassociated proteins in SNUC5/5FUR cells upon 5-FU treatment, we assessed the phosphorylation of p38, JNK, p53, and ATM. Pretreatment with si-GRP78 induced a significant increase in the phosphorylation of oxidative stress-associated proteins in SNUC5/5FUR cells after treatment with 5-FU (Figure 3C-E). These findings indicate that GRP78 controls intracellular ROS production upon 5FU treatment by regulating the phosphorylation of oxidative stress-associated proteins.

GRP78 activates the AKT pathway following 5-FU treatment. The AKT pathway plays a pivotal role in regulating cell survival signals, as well as apoptosis-inducing factors (14). We investigated whether GRP78 is involved in 5-FU-mediated apoptosis by regulating the AKT pathway. For this, we analyzed the phosphorylation of PI3K, AKT, and mTOR in 5FU-treated SNUC5/5FUR cells (Figure 4A). Pretreatment with si-GRP78 significantly reduced the phosphorylation of PI3K, AKT, and mTOR in 5-FU-treated SNUC5/5FUR cells (Figure 4B). The phosphorylation of PI3K and AKT significantly decreased in si-GRP78-transfected SNUC5/5FUR cells compared to control SNUC5/5FUR cells (Figure 4B). These results reveal that GRP78 is involved in the inhibition of 5FU-induced apoptosis, by regulating the PI3K-AKT-mTOR signaling pathway.

GRP78 is involved in the proliferation of 5-FU-resistant CRC cells. To explore the effect of GRP78 on the proliferation of 5-FU-resistant CRC cells, the expression of cell cycle-associated proteins CDK2, cyclin E, CDK4, and cyclin D1 were assessed in SNUC5/5FUR cells (Figure 5A). Treatment with 5-FU significantly reduced the levels of CDK2, cyclin E, CDK4, and cyclin D1 in SNUC5/5FUR cells (Figure 5B). In addition, pretreatment with si-GRP78 induced a significant decrease in the expression of these proteins in 5-FU-treated SNUC5/5FUR cells (Figure 5B). Furthermore, flow cytometric analysis of PI-stained 5-FUtreated SNUC5/5FUR cells showed that GRP78 knockdown led to a significant decrease in the proportion of cells in the S-phase (Figure 5C and D). These results indicate that 

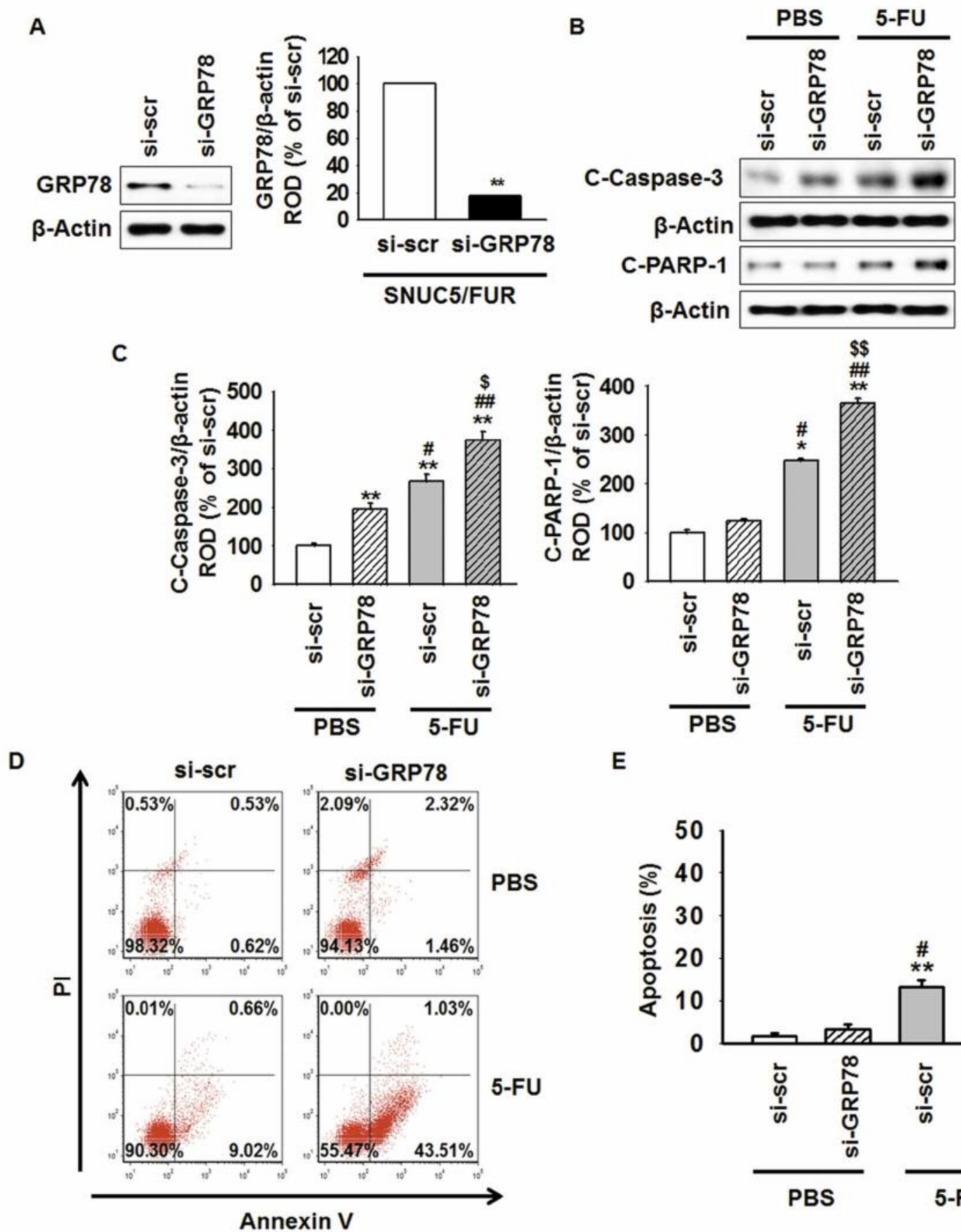

E

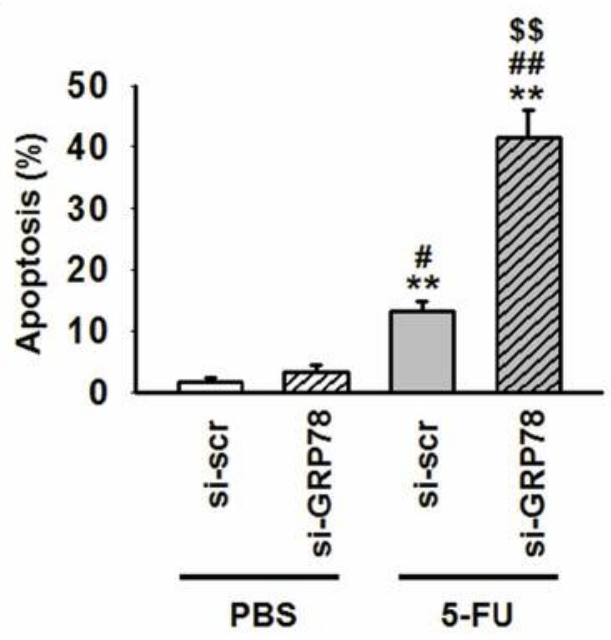

Figure 2. Effect of glucose-regulated protein (GRP78) on 5-fluorouracil (5-FU)-induced apoptosis of and 5-fluorouracil-resistant (SNUC5/5FUR) colorectal cancer cells. A: Western blot analysis of GRP78 in SNUC5/5FUR cells after transfection with scrambled (si-scr) or GRP78 siRNA (siGRP78). The expression of GRP78 was normalized relative to that of $\beta$-actin. Values represent the mean \pm standard error of the mean (SEM). **Significantly different at $p<0.01$ compared si-scr. B,C: Western blot and densitometric analysis of cleaved caspase-3 (C-caspase-3) and cleaved poly(ADP ribose)-polymerase 1 (C-PARP1) expression after transfection of SNUC5/5FUR cells with si-scr or si-GRP78, followed by treatment with phosphate-buffered saline (PBS, control) or $5-F U(140 \mu M)$ for 48 h. Significantly different at $* p<0.05$ and $* * p<0.01$ compared to PBS-treated SNUC5/5FUR cells pretreated with si-scr; ${ }^{\#} p<0.05$ and ${ }^{\# \#} p<0.01$, compared with PBS-treated SNUC5/5FUR cells pretreated with si-GRP78; $\$ p<0.05$ and $\$ \$ p<0.01$ compared to 5-FU-treated SNUC5/5FUR cells pretreated with si-scr. D: Apoptosis was measured by flow cytometry using propidium iodide (PI) and annexin V-fluorescein isothiocyanate staining. E: Percentage of apoptotic cells in different treatment groups. Values represent the mean \pm SEM. Significantly different at $* * p<0.01$ compared to PBS-treated SNUC5/5FUR cells pretreated with si-scr; ${ }^{\#} p<0.05$ and ${ }^{\# \# p<0.01 \text { compared }}$ with PBS-treated SNUC5/5FUR cells pretreated with si-GRP78; $\$ \$ p<0.01$ compared to 5-FU-treated SNUC5/5FUR cells pretreated with si-scr. The expression levels were determined by densitometry, and normalized relative to the expression level of $\beta$-actin. 
A
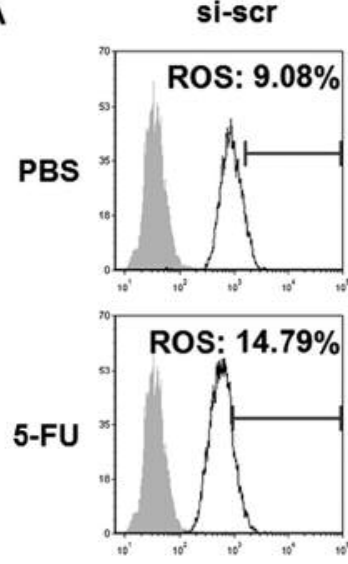

si-GRP78
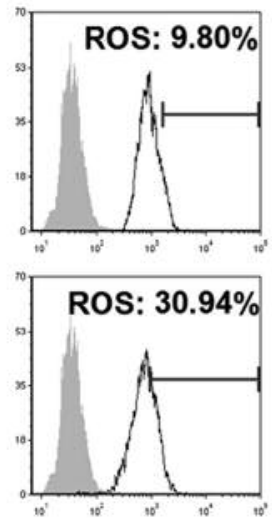

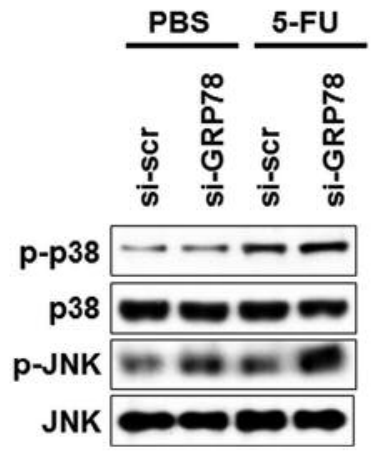

C

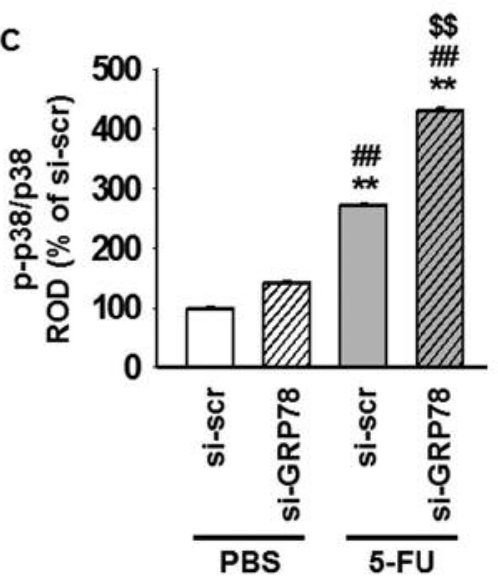

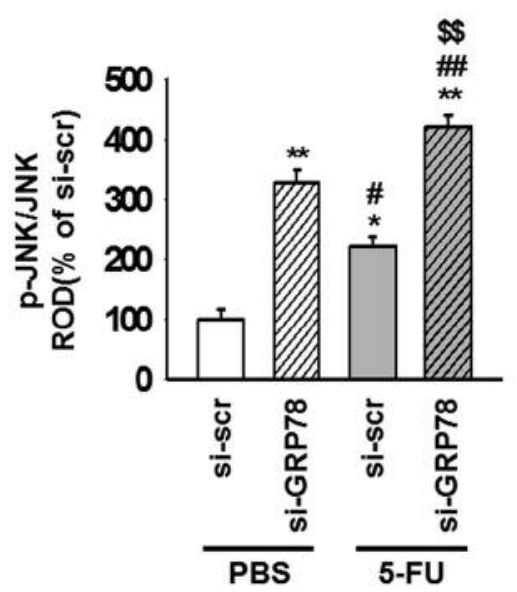

D

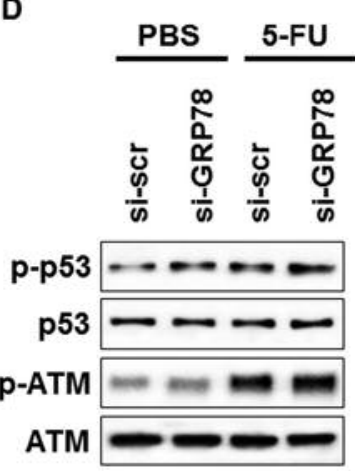

E

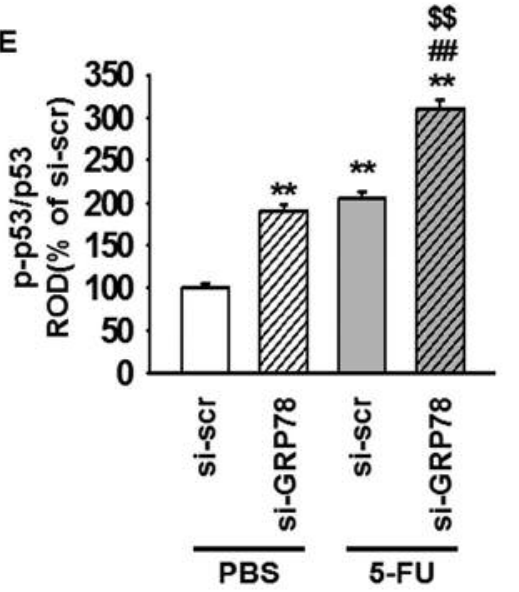

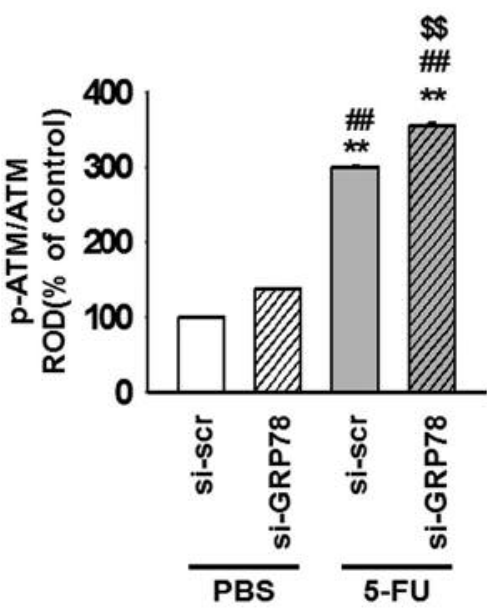

Figure 3. Glucose-regulated protein (GRP78) regulates reactive oxygen species (ROS) production and stress-associated signaling pathway components in 5-fluorouracil (5-FU)-treated 5-fluorouracil-resistant (SNUC5/5FUR) colorectal cancer cells. A: Flow cytometric analysis of dihydroethidium-stained cells to detect intracellular ROS production in SNUC5/5FUR cells pretreated with scrambled (si-scr) or GRP78 siRNA (si-GRP78), and subsequently treated with 5-FU for $48 \mathrm{~h}$. Grey lines indicate the negative control. Black lines indicate the cells stained with dihydroethidium. B, C: Western blot and densitometric analysis illustrating the levels of phosphorylated p38 (p-p38) and c-JUN N-terminal kinase (p-JNK) in SNUC5/5FUR cells transfected with si-scr or si-GRP78 and subsequently treated with phosphate-buffered saline (PBS) or 5-FU (140 $\mu M)$ for 48 h. Significantly different at *p<0.05 and ${ }^{* *} p<0.01$ compared to PBS-treated SNUC5/5FUR cells pretreated with si-scr; ${ }^{\#} p<0.05$ and ${ }^{\# \#} p<0.01$ compared to PBS-treated SNUC5/5FUR cells pretreated with si-GRP78; $\$ \$ p<0.01$ compared with 5-FU-treated SNUC5/5FUR cells pretreated with si-scr. D, E: Western blots and densitometric analysis showing the expression levels of phosphorylated 553 (p-p53) and ataxia-telangiectasia mutated (p-ATM) in SNUC5/5FUR cells transfected with si-scr or si-GRP78 and subsequently treated with PBS or 5-FU $(140 \mu M)$ for 48 h. Significantly different at $p<0.01$ compared to PBS-treated SNUC5/5FUR cells pretreated with **si-scr; or \#\#si-GRP78; $\$ \$ p<0.01$ compared to 5-FU-treated SNUC5/5FUR cells pretreated with si-scr. The expression levels were determined by densitometry, and normalized relative to the expression level of $\beta$-actin. Values represent the mean \pm SEM. 
A

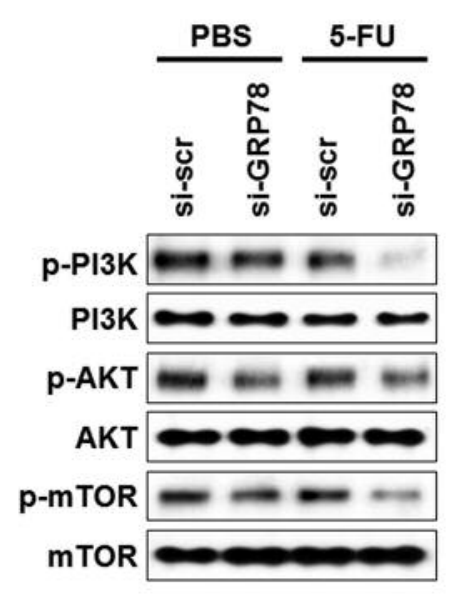

B
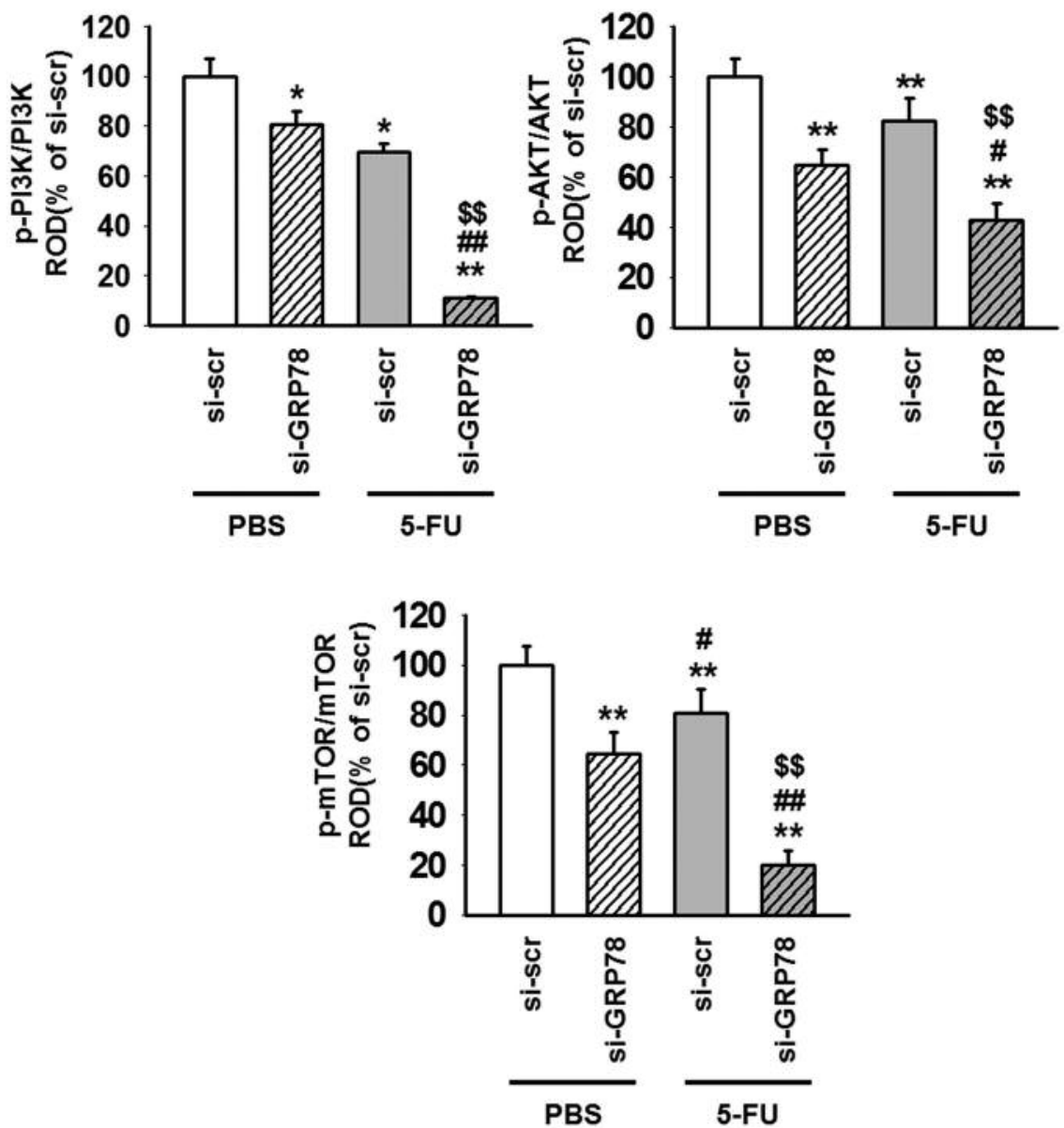

Figure 4. Glucose-regulated protein (GRP78) regulates phosphatidylinositol 3-kinase-AKT-mammalian target of rapamycin (PI3K-AKT-mTOR) signaling axis in 5-fluorouracil (5-FU)-treated 5-fluorouracil-resistant (SNUC5/5FUR) colorectal cancer cells. A, B: Western blot and densitometric analysis for analyzing the expression of phosphorylated PI3K (p-PI3K), AKT ( $p$-AKT), and mTOR (p-mTOR) in SNUC5/5FUR cells transfected with scrambled (si-scr) or GRP78 siRNA (si-GRP78), and subsequently treated with phospho buffered saline (PBS) or 5-FU (140 $\mu M)$ for 48 h. The expression levels of $p$-PI3K, $p$-Akt, and p-mTOR were determined by densitometry and normalized relative to the expression level of $\beta$-actin. Values represent the mean \pm SEM. Significantly different at $* p<0.05$ and ${ }^{* *} p<0.01$ compared to PBS-treated SNUC5/5FUR cells pretreated with si-scr; ${ }^{\#} p<0.05$ and ${ }^{\# \#} p<0.01$ compared to PBS-treated SNUC5/5FUR cells pretreated with si-GRP78; $\$ \$ p<0.01$ compared with 5-FU-treated SNUC5/5FUR cells pretreated with si-scr.

GRP78 is involved in the proliferation of 5-FU-resistant CRC cells by regulating the expression of cell cycleassociated proteins.

\section{Discussion}

In this study, we showed that the expression of GRP78 was high in 5-FU-resistant CRC cells, resulting in the augmentation of cell survival and proliferation post-5-FU treatment. In addition, GRP78 controlled the production of ROS in response to 5-FU-mediated oxidative stress. Knockdown of GRP78 induced apoptosis and inhibited proliferation of SNUC5/5FUR cells by regulating the apoptosis-, survival-, and proliferation-associated signaling pathways.

GRP78 is known to be highly expressed in tumor cells. It plays an important role in tumor cell proliferation, angiogenesis, metastasis, and resistance to anticancer drugs (15). In particular, GRP78 is involved in the development of therapeutic resistance in several types of cancer cell. High expression of GRP78 regulates anti-estrogen resistance in breast cancer cells (16). Elevated levels of GRP78 are also associated with trastuzumab and doxorubicin resistance in breast cancer cells (17). In ovarian cancer cells, GRP78 had 


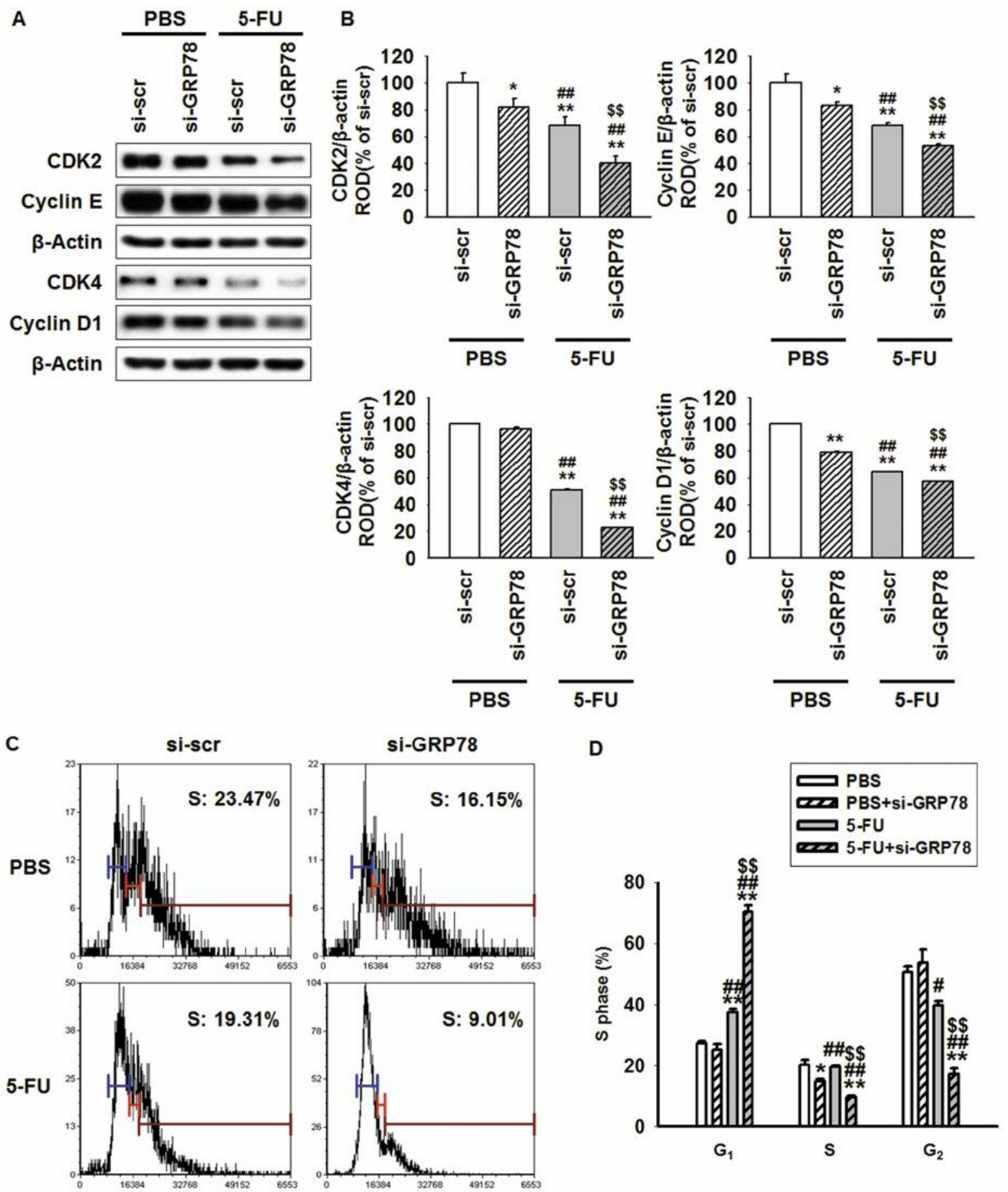

Figure 5. Glucose-regulated protein (GRP78) is involved in proliferation of 5-fluorouracil (5-FU)-treated 5-fluorouracil-resistant (SNUC5/5FUR) colorectal cancer cells via regulation of cell cycle-associated proteins. A, B: Western blot and densitometric analysis for analyzing the levels of cyclin-dependent kinase 2 (CDK2), cyclin E, CDK4, and cyclin D1 in SNUC5/5FUR cells transfected with scrambled (si-scr) or GRP78 siRNA (siGRP78), and subsequently treated with phospho buffered saline (PBS) or 5-FU (140 $\mu M)$ for 48 h. Significantly different at *p<0.05 and **p<0.01 compared with PBS-treated SNUC5/5FUR cells pretreated with si-scr; \#\#p<0.01 compared to PBS-treated SNUC5/5FUR cells pretreated with siGRP78; $\$ \$ p<0.01$ compared to 5-FU-treated SNUC5/5FUR cells pretreated with si-scr. The expression levels were determined by densitometry, and normalized relative to the expression level of $\beta$-actin. $C, D$ : Flow cytometric analysis for PI staining to assess $G_{0} / G_{1}, S$, and $G_{2} / M$ phase populations in SNUC5/5FUR cells treated with si-scr or si-GRP78, and subsequently treated with 5-FU. Significantly. different at $* p<0.05$ and $* * p<0.01$ compared with PBS-treated SNUC5/5FUR cells pretreated with si-scr; \#\# $p<0.01$ compared with PBS-treated SNUC5/5FUR cells pretreated with si-GRP78; ${ }^{\$} p<0.01$ compared with 5-FU-treated SNUC5/5FUR cells pretreated with si-scr. Values represent the mean \pm SEM. 
an anti-senescence effect against cisplatin treatment via regulation of the ATM pathway and induction of calcium efflux from the ER to the cytoplasm (18). These findings suggest that GRP78 could be targeted for overcoming the problem of drug resistance in cancer cells.

Recent studies have suggested that an elevated GRP78 level can protect cancer cells from the cytotoxic effects of chemotherapeutic agents, such as doxorubicin, taxol, and 5-FU $(8,19,20)$. We observed that GRP78 knockdown resulted in the increase of apoptosis of 5-FU-treated SNUC5/5FUR cells via activation of the apoptosis-associated signaling proteins, such as caspase- 3 and PARP1. In addition, GRP78 regulated the production of intracellular ROS by modulating the stressassociated signaling pathway proteins, including p38, JNK, p53, and ATM. In Chinese hamster ovary, human leukemia, and bladder carcinoma cell lines, GRP78 overexpression induced resistance to cytotoxic drugs (such as topoisomerase inhibitors) and induced apoptosis via suppression of caspase-7 activation (7). In the DLD-1 colon cancer cells, GRP78 knockdown enhanced apoptosis and intracellular ROS production via down-regulation of the AKT pathway and activation of protein phosphatase $2 \mathrm{~A}$, following epirubicin treatment (21). In glial cells, GRP78 enhanced cellular antioxidant levels through suppression of lipid peroxidation, following hydrogen peroxide exposure (22). These findings indicate that 5-FU-mediated apoptosis is regulated by GRP78. This suggests that 5-FU-induced increase in GRP78 expression results in the inhibition of apoptosis in 5-FU resistant CRC cells via regulation of intracellular ROS production.

The PI3K-AKT-mTOR signaling axis plays an important role in cell survival and growth under various physiological and pathological conditions (23). In particular, this pathway plays a key role in regulating cancer biology, since cancer cells usually reside in harsh environments, such as with low oxygen, $\mathrm{pH}$, and nutrient supply (23). Our results demonstrated that the PI3K-AKT-mTOR signaling pathway was activated in SNUC5/5FUR cells and knockdown of GRP78 reduced the phosphorylation of PI3K, AKT, and mTOR. GRP78 acts as a co-receptor or signal-transducing receptor for soluble ligands, including $\alpha 2$-macroglobulin, tumor differentiation factor, vaspin, T-cadherin, and Cripto (24). The binding of GRP78 to these ligands promotes the activation of the PI3K-AKT pathway, resulting in increased cell survival, adhesion, and migration (24). The resistance of breast and prostate cancer cells to chemotherapy is associated with the localization of GRP78 to the cell surface, where it binds to PI3K, which is an activator of AKT (25). Overexpression of GRP78 enhances the formation of phosphatidylinositol (3,4,5)-trisphosphate (PIP3), which is present downstream of PI3K; however, mutation of the $N$-terminal region of GRP78 reduces the binding of GRP78 to PI3K and PIP3 (25). In prostate cancer cells, blocking of cell surface GRP78 by anti-GRP78 reduced the growth of cells via inhibition of the PI3K-AKT pathway (26). In addition, GRP78 promoted breast cancer cell survival via activation of mTORregulated pro-survival autophagy. Our results suggest that the 5-FU-induced increase in GRP78 expression promotes survival of 5-FU resistant CRC cells via regulation of the PI3K-AKTmTOR signaling axis.

We also revealed that the 5-FU-induced increase in GRP78 expression increased the proliferation of SNUC5/5FUR cells; however, GRP78 knockdown inhibited cell proliferation by regulating expression of cell cycle-associated proteins CDK2, cyclin E, CDK4, and cyclin D1. GRP78 was found to enhance cell proliferation and survival during mouse embryonic development (27). Overexpression of GRP78 increased cell proliferation in chondrocyte development by enhancement of the S-phase population (28). In breast cancer cells, GRP78 facilitated cell proliferation through activation of the Janus kinase 2/signal transducer and activator of transcription 3 pathway (29). In addition, inhibition of GRP78 and GRP94 reduced cellular proliferation in a human gastric cancer cell line (30). These findings suggest that the 5-FU-induced increase in GRP78 expression plays a key role in the development of 5-FU resistance in CRC cells.

Taken together, our findings demonstrate that drug-induced expression of GRP78 prevents apoptosis and production of intracellular ROS. It also enhances cell survival and proliferation of drug-resistant CRC cells via regulation of the apoptosis-, survival-, and cell cycle-associated signaling pathways. These findings indicate that GRP78 could be an important biomarker for drug-resistant CRC. In conclusion, the development of resistance in colon cancer cells against chemotherapeutic agents might be combated by targeting GRP78. This may be used as a novel strategy for treating colon cancer.

\section{Conflicts of Interest}

The Authors have no conflicts of interest to declare with regards to this study.

\section{Acknowledgements}

This work was supported by the Soonchunhyang University Research Fund, a National Research Foundation grant funded by the Korean government [grant number NRF-2016R1D1A3B01007727, NRF-2017M3A9B4032528] The funding agencies had no role in formulating the study design, data collection or analysis, the decision to publish, or preparation of the article.

\section{References}

1 Siegel RL, Miller KD and Jemal A: Cancer Statistics, 2017. CA Cancer J Clin 67: 7-30, 2017.

2 Holohan C, Van Schaeybroeck S, Longley DB and Johnston PG: Cancer drug resistance: an evolving paradigm. Nat Rev Cancer 13: 714-726, 2013.

3 Lee AS: Glucose-regulated proteins in cancer: molecular mechanisms and therapeutic potential. Nat Rev Cancer 14: 263276, 2014. 
4 Wang M, Wey S, Zhang Y, Ye R and Lee AS: Role of the unfolded protein response regulator GRP78/BiP in development, cancer and neurological disorders. Antioxid Redox Signal 11: 2307-2316, 2009

5 Gonzalez-Gronow M, Selim MA, Papalas J and Pizzo SV: GRP78: a multifunctional receptor on the cell surface. Antioxid Redox Signal 11: 2299-2306, 2009.

6 Erhardt P, Schremser EJ and Cooper GM: B-RAF inhibits programmed cell death downstream of cytochrome $\mathrm{c}$ release from mitochondria by activating the MEK/ERK pathway. Mol Cell Biol 19: 5308-5315, 1999.

7 Reddy RK, Mao C, Baumeister P, Austin RC, Kaufman RJ and Lee AS: Endoplasmic reticulum chaperone protein GRP78 protects cells from apoptosis induced by topoisomerase inhibitors: role of ATP binding site in suppression of caspase-7 activation. J Biol Chem 278: 20915-20924, 2003.

8 Pyrko P, Schonthal AH, Hofman FM, Chen TC and Lee AS: The unfolded protein response regulator GRP78/BiP as a novel target for increasing chemosensitivity in malignant gliomas. Cancer Res 67: 9809-9816, 2007.

9 Langer R, Feith M, Siewert JR, Wester HJ and Hoefler H: Expression and clinical significance of glucose regulated proteins GRP78 (BiP) and GRP94 (GP96) in human adenocarcinomas of the esophagus. BMC Cancer 8: 70, 2008.

10 Mhaidat N, Jaradat S, Aldaher A and Ghabkari A: Inhibition of GRP78 sensitizes colorectal cancer cells to paclitaxel-induced apoptosis by activation of caspase-4. Int J Biol Biomed Eng 5: 41-48, 2011.

11 Longley DB, Harkin DP and Johnston PG: 5-fluorouracil: mechanisms of action and clinical strategies. Nat Rev Cancer 3: 330-338, 2003.

12 Walther A, Johnstone E, Swanton C, Midgley R, Tomlinson I and Kerr D: Genetic prognostic and predictive markers in colorectal cancer. Nat Rev Cancer 9: 489-499, 2009.

13 Simon HU, Haj-Yehia A and Levi-Schaffer F: Role of reactive oxygen species (ROS) in apoptosis induction. Apoptosis 5: 415$418,2000$.

14 Clerkin JS, Naughton R, Quiney C and Cotter TG: Mechanisms of ROS modulated cell survival during carcinogenesis. Cancer Lett 266: 30-36, 2008.

15 Luo B and Lee AS: The critical roles of endoplasmic reticulum chaperones and unfolded protein response in tumorigenesis and anticancer therapies. Oncogene 32: 805-818, 2013.

16 Cook KL, Shajahan AN, Warri A, Jin L, Hilakivi-Clarke LA and Clarke R: Glucose-regulated protein 78 controls cross-talk between apoptosis and autophagy to determine antiestrogen responsiveness. Cancer Res 72: 3337-3349, 2012.

17 Cook KL and Clarke R: Role of GRP78 in promoting therapeutic-resistant breast cancer. Future Med Chem 7: 15291534, 2015.
18 Li W, Wang W, Dong H, Li Y, Li L, Han L, Han Z, Wang S, Ma $\mathrm{D}$ and Wang H: Cisplatin-induced senescence in ovarian cancer cells is mediated by GRP78. Oncol Rep 31: 2525-2534, 2014.

19 Lee E, Nichols P, Spicer D, Groshen S, Yu MC and Lee AS: GRP78 as a novel predictor of responsiveness to chemotherapy in breast cancer. Cancer Res 66: 7849-7853, 2006.

20 Wang J, Yin Y, Hua H, Li M, Luo T, Xu L, Wang R, Liu D, Zhang Y and Jiang Y: Blockade of GRP78 sensitizes breast cancer cells to microtubules-interfering agents that induce the unfolded protein response. J Cell Mol Med 13: 3888-3897, 2009.

21 Chang YJ, Huang YP, Li ZL and Chen CH: GRP78 knockdown enhances apoptosis via the down-regulation of oxidative stress and AKT pathway after epirubicin treatment in colon cancer DLD-1 cells. PloS one 7: e35123, 2012.

22 Suyama K, Watanabe M, Sakabe K, Otomo A, Okada Y, Terayama H, Imai T and Mochida J: GRP78 suppresses lipid peroxidation and promotes cellular antioxidant levels in glial cells following hydrogen peroxide exposure. PloS one 9: e86951, 2014.

23 Porta C, Paglino C and Mosca A: Targeting PI3K/AKT/mTOR signaling in cancer. Front Oncol 4: 64, 2014.

24 Casas C: GRP78 at the centre of the stage in cancer and neuroprotection. Front Neurosci 11: 177, 2017.

25 Zhang Y, Tseng CC, Tsai YL, Fu X, Schiff R and Lee AS: Cancer cells resistant to therapy promote cell surface relocalization of GRP78 which complexes with PI3K and enhances PI(3,4,5)P3 production. PloS One 8: e80071, 2013.

26 Misra UK and Pizzo SV: Modulation of the unfolded protein response in prostate cancer cells by antibody-directed against the carboxyl-terminal domain of GRP78. Apoptosis 15: 173-182, 2010 .

27 Luo S, Mao C, Lee B and Lee AS: GRP78/BiP is required for cell proliferation and protecting the inner cell mass from apoptosis during early mouse embryonic development. Mol Cell Biol 26: 5688-5697, 2006.

28 Xiong Z, Jiang R, Li X, Liu Y and Guo F: Different Roles of GRP78 on cell proliferation and apoptosis in cartilage development. Int J Mol Sci 16: 21153-21176, 2015.

29 Yao X, Liu H, Zhang X, Zhang L, Li X, Wang C and Sun S: Cell surface GRP78 accelerated breast cancer cell proliferation and migration by activating STAT3. PloS one 10: e0125634, 2015.

30 Zhang X, Zhang L, Wang S, Wu D and Yang W: Decreased functional expression of Grp78 and Grp94 inhibits proliferation and attenuates apoptosis in a human gastric cancer cell line in vitro. Oncol Lett 9: 1181-1186, 2015.

Received July 11, 2017

Revised July 27, 2017

Accepted August 1, 2017 